\title{
Hybrid white light emitting devices (HWLEDs) from organic polymer and PbS nanocrystals by multiple excitons
}

\author{
AKEEL M. KADIM* \\ Medical Physics, College of Science, Al Karkh University of Science, Iraq
}

\begin{abstract}
Hybrid white light-emitting devices (HWLEDs) were fabricated using FTO/PEDOT: PSS/PbS/Alq 3 /Ni system and synthesized by phase separation process. In the present study, the multiple excitons generation in lead sulfide (PbS) NCs, which is characteristic of PbS NCs, was used to induce an effective and regulated energy transfer to an HWLED. The HWLED consisted of three layers successively deposited on FTO glass substrate; the first layer consisted of poly (3,4-ethylenedioxythiophene) polystyrene sulfonate (PEDOT: PSS) blended with polymethyl methacrylate (PMMA) organic polymer in the 1:1 ratio, while the second layer consisted of PbS NCs. Finally, above the layer of the PbS NCs, Tris (8-hydroxyquinoline) aluminum (Alq 3 ) layer was deposited. The white light was generated with quite a good efficiency due to the confinement effect that makes the energy gap greater. The characteristics of the current-voltage (I-V) indicate acceptable conditions for the generation of white light by multiple excitons. It was found that the emission levels able to produce white luminescence, classified based on the coordinate system of chromaticity (CIE 1931), are $\mathrm{x}=0.31, \mathrm{y}=0.33$ while the correlated color temperature (CCT) is about $6250 \mathrm{~K}$. The HWLEDs made from PbS NCs with hole injection from the organic polymer (PEDOT: PSS with PMMA), and electron injection from organic molecules $\left(\mathrm{Alq}_{3}\right)$ are capable of white light generation.
\end{abstract}

Keywords: organic devices; HWLEDs; PbS; PEDOT: PSS; $\mathrm{Alq}_{3}$

\section{Introduction}

Because of their unique advantages, such as self-emission, durability, clarity, low cost, and high performance, possibility of using as a surface light source, hybrid white light-emitting devices (HWLEDs) have attracted great interest, suggesting their wide applications in flat-panel, full-color displays and solid-state lighting environments [1]. Extensive work has been done in recent years on HWLEDs to achieve high system performance, color rendering index (CRI), and color strength needed for lighting applications. Many articles reported WLEDs with an external quantum efficiency (EQE) close to $20 \%$. This is also considered as a statistical maximum limit value for LEDs in flatpanel systems [1, 2]. Rapid development of light sources revealed the need for LEDs with improved shape, capacity and stability, enabling fabrication of space-saving light sources. Furthermore, largescale solutions and cost-effective manufacturing

*E-mail: akeel_a86@yahoo.com techniques can open the way to new areas of applications and market strategies, such as disposable electronics or smart materials [3, 4]. A general reason for investigation and improvement of semiconductor quantum dots results from the fact that they are universal technically tunable devices for lighting and sensing applications [4].

$\mathrm{PbS}$ NCs, belonging to the IV-VI semiconductors, is a highly interesting material for processing with sodium chloride crystal structure, having a temperature-dependent direct bandgap of approximately $0.29 \mathrm{eV}$. It is useful for sensing infrared and visible light $[4,5]$. Light-emitting applications are the key field of the studies in which the $\mathrm{PbS}$ nanocrystals are usually used in a form of colloidal $\mathrm{PbS}$ quantum dots. Similar to $\mathrm{PbSe}, \mathrm{PbS}$ has high photosensitivity in the light-absorbing range, from the visible to near-infrared, due to the bandgap of $\mathrm{PbS}$ which can be adjusted in the NIR region. The PbS colloidal NCs generate also multiple excitons. Both these properties make the PbS NCs HWLEDs the best candidate for light generation devices $[6,7]$. There is a requirement to build new 
hybrid WLEDs with improved system architecture and to improve production techniques to obtain superior product at low cost and high yield. Produced HWLEDs should be able to compete with traditional illumination and display techniques $[8,9]$.

Herein, a new method has been developed for direct synthesizing of $\mathrm{PbS} \mathrm{NCs}$ as well as lightemitting device for white light applications. This work provides an overview on HWLED prepared using $\mathrm{PbS}$ colloidal NCs with the efficiency enhanced by multiple exciton generation (MEG) process. This process was used to improve the effectiveness of the HWLED.

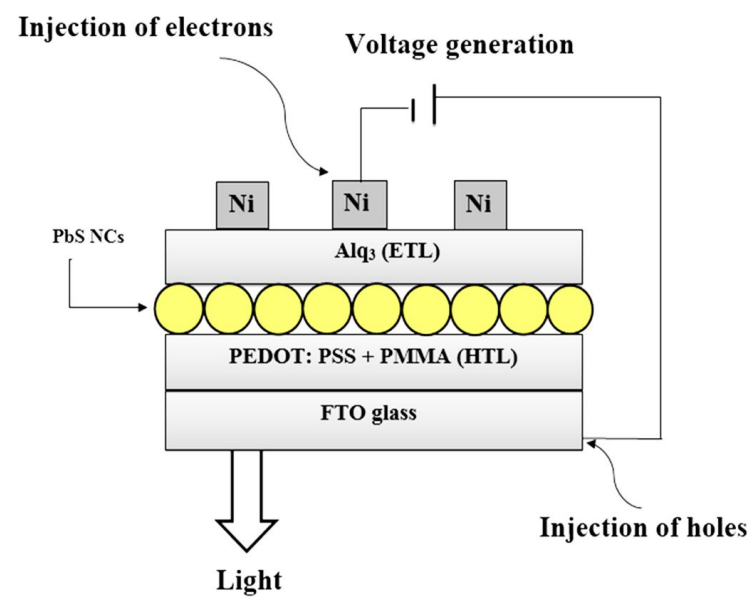

Fig. 1. Typical structure of a hybrid white light-emitting device (HWLED).

\section{Experimental}

All the primary chemicals were supplied by Fluka Company and used without further purification, while SigmaAldrich Chemie GmbH (Germany) supplied polystyrene sulfonate (PEDOT:PSS) and Tris (8-hydroxyquinoline) aluminum ( $\left.\mathrm{Alq}_{3}\right)$ with poly(3,4ethylenedioxythiophene). The lead sulfide $\mathrm{PbS} \mathrm{NCs} \mathrm{in} \mathrm{the} \mathrm{colloidal} \mathrm{form} \mathrm{were} \mathrm{prepared}$ from 0.1 M sodium sulfide $\left(\mathrm{Na}_{2} \mathrm{~S}\right)$ and $0.1 \mathrm{M}$ lead chloride $\left(\mathrm{PbCl}_{2}\right)$ at 1:2 mole ratio. $0.32 \mathrm{~g}$ of lead chloride $\mathrm{PbCl}_{2}$ in powder form was dissolved in $10 \mathrm{ml}$ of distilled water and $0.24 \mathrm{~g}$ of sodium sulfide powder $\left(\mathrm{Na}_{2} \mathrm{~S}\right)$ was dissolved in $10 \mathrm{ml}$ of distilled water. The suspensions $\left(\mathrm{PbCl}_{2}\right.$ and
$\mathrm{Na}_{2} \mathrm{~S}$ ) were mixed for about 30 minutes in a 3-neck flask at $25{ }^{\circ} \mathrm{C}$ under the flow of argon gas until the color was changed from light black to dark brown. This shows that $\mathrm{PbS}$ nanostructures have developed. The developing of $\mathrm{PbS} \mathrm{NCs}$ was possible due to addition of isopropanol and 25-minute centrifugation.

The light-emitting device (LED) production process consisted of several phases. First, $70 \mathrm{mg} / \mathrm{ml}$ of PEDOT: PSS was dissolved and mixed with polymethyl methacrylate (PMMA) in 1:1 ratio (PMMA was used to prevent cracks) in $1 \mathrm{ml}$ of chloroform; Moreover, $70 \mathrm{mg} / \mathrm{ml}$ of $\mathrm{Alq}_{3}$ was dissolved in $3 \mathrm{ml}$ of chloroform. Finally, the layers were sequentially deposited onto FTO substrate as in Fig. 1.

Fig. 1 illustrates the fabricated organic white light-emitting device. The system consists of three layers, which were sequentially deposited on the FTO glass substrate. The FTO glass substrates were cleaned successively using detergent, acetone, isopropyl alcohol, and deionized water before the deposition process at $2000 \mathrm{rpm}$ for around $10 \mathrm{~s}$ to 15 s concerning each coating. The first layer - PEDOT: PSS was mixed with PMMA in the ratio 1:1, while the second layer consisted of PbS NCs. Once each coating was applied, it was dried in an oven at $80{ }^{\circ} \mathrm{C}$ for $15 \mathrm{~min}$. Then, the $\mathrm{Alq}_{3}$ layer was deposited over the layer of PbS NCs. The film thickness was determined using Tolansky method (interference method) The thicknesses of PEDOT: PSS with PMMA and $\mathrm{Alq}_{3}$ layers were $40 \mathrm{~nm}$ and $32 \mathrm{~nm}$, respectively, while the $\mathrm{PbS}$ NC layer thickness was $20 \mathrm{~nm}$. FTO layer thickness and its sheet resistance were $100 \mathrm{~nm}$ and $8 \Omega / \mathrm{sq}$, respectively. Nickel (Ni) was coated on the hybrid film afterward to obtain the cathode with a thickness of $200 \mathrm{~nm}$.

\section{Results and discussion}

UV-Vis spectra are commonly used to study semiconductor NCs. When the particle size decreases, the absorption wavelength $\left(\lambda_{\max }\right)$ can change to shorter wavelength, as the bandgap increases for the particles that are nanosized. It results from the effect of quantum confinement in semiconductor nanocrystals. The absorption and 
photoluminescence (PL) spectra for PbS NCs are shown in Fig. 2.

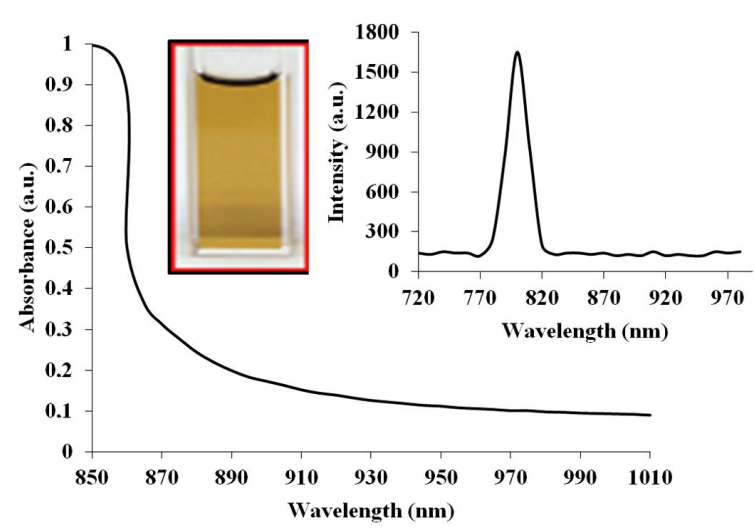

Fig. 2. Absorption and PL spectra of PbS NCs.

The absorption of PbS NCs increases as its surface state level increases. All surface state levels and bandgap may be increased when particle size is reduced to nanoscale dimensions. The absorption spectrum of $\mathrm{PbS}$ NCs reveals a strong absorption in the near-infrared region (NIR). Such finding is in a strong agreement with the absorption spectra reported by other researchers $[10,11]$. The PL of $\mathrm{PbS}$ NCs in Fig. 2 shows that the band edge transmission is centered around $800 \mathrm{~nm}$ and the other peaks can be ascribed to the development of surface levels in the energy gap suggested by other researches $[12,13]$. The energy gap of $\mathrm{PbS} \mathrm{NCs}$ determined from the PL spectra using the relationship: $E=1240 / \lambda(\mathrm{nm})$ was found to be nearly $1.55 \mathrm{eV}$. The quantum confinement effect occurs in nanomaterials with nanometric sizes and especially affects the electronic properties. Thus, the special attention was given to preventing the aggregation of $\mathrm{PbS}$ NCs to enhance their optical properties.

Fig. 3 shows the surface morphology of $\mathrm{PbS}$ NCs. The NCs thin film deposited on the glass substrate was subjected to SEM investigation. The surface morphology of the prepared PbS NCs was observed at $30 \mathrm{kX}$ magnification, as shown in Fig. 3. The SEM image and the histogram of particle size distribution of the PbS NCs were analyzed by using ImageJ software. The average grain size was estimated to be around $16 \mathrm{~nm}$. Fig. 3 shows that the shapes of the NCs are almost spherical, and the image doesn't show any aggregation of NCs within $20 \mathrm{~nm}$.
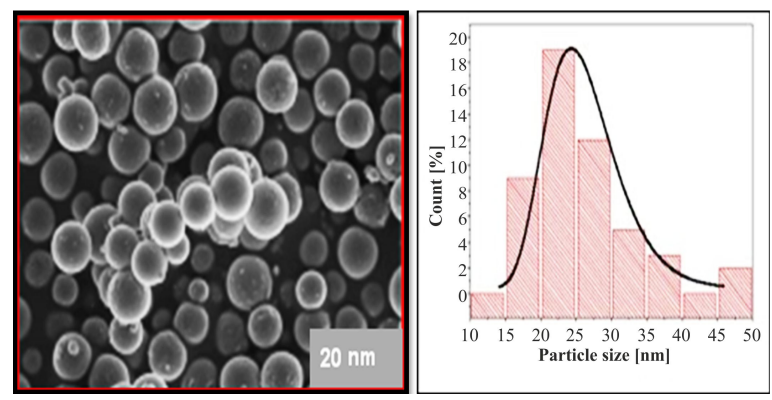

Fig. 3. Scanning electron microscope (SEM) image and histogram of size distribution of PbS NCs.

The I-V characteristics of HWLEDs of the fabricated $\mathrm{FTO} / \mathrm{PEDOT}$ : $\mathrm{PSS} / \mathrm{PbS} / \mathrm{Alq}_{3} / \mathrm{Ni}$ are shown in Fig. 4. In general, the white light emission was observed in the current range of $0.02 \mathrm{~mA}$ to $1 \mathrm{~mA}$.



Fig. 4. I-V characteristics of FTO/PEDOT:PSS/PbS/ $\mathrm{Alq}_{3} / \mathrm{Ni}$.

The current of the HWLEDs I-V characteristics increases exponentially with voltage as a result of reduced thickness of the depletion layer. The barrier at the forward bias is reduced due to the exponential growth of multiple electrons and holes inside the conductive and valence bands, thereby the current increases at the forward bias [14-17].

Fig. 5 shows the electroluminescence spectrum of FTO/PEDOT: PSS/PbS/Alq $/ \mathrm{Ni}$ at the forward bias voltage less than $4 \mathrm{~V}$. This voltage is called "operating voltage" for the light generated obtained from the studies of FTO/PEDOT: PSS/PbS/Alq $3 / \mathrm{Ni}$ 
HWLED emission which were performed using a photomultiplier detector at room temperature.

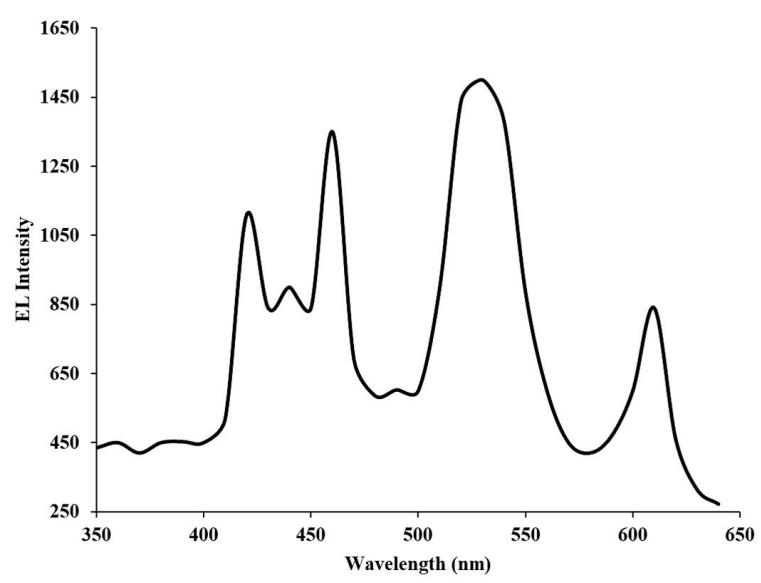

Fig. 5. Electroluminescence spectrum of FTO/PEDOT: $\mathrm{PSS} / \mathrm{PbS} / \mathrm{Alq}_{3} / \mathrm{Ni}$

Fig. 5 demonstrates the electroluminescence emission spectrum of FTO/PEDOT: $\mathrm{PSS} / \mathrm{PbS} / \mathrm{Alq}_{3} / \mathrm{Ni}$ device measured at a forward bias voltage less than $4 \mathrm{~V}$, indicating that the white light is produced. Fig. 5 shows the peaks at $460 \mathrm{~nm}$, $530 \mathrm{~nm}$ and $610 \mathrm{~nm}$ and the extra peaks caused by defect levels. The carrier transport mechanism in the HWLEDs is that, the PEDOT: PSS serves as a hole transporting material and it contributes to an increase in the emission intensity of hybrid devices [18-21]. The holes are injected from the FTO anode into the high occupied molecular orbital (HOMO) of the PEDOT: PSS with PMMA and electrons are injected into the conduction band from the nickel $(\mathrm{Ni})$ cathode; these electrons are again injected into $\mathrm{Alq}_{3}$ layer, which is used as an electron transporting material and emission layer. The number and the mobility of electrons increase towards the PbS NCs layer [22-24]. Thus, the hole and electron pairs in PbS NCs layer cause the generation of multiple excitons (MEG) in the band-to-band recombination. The recombination processes of holes and electrons of various wavelengths due to the defect sates result in emission of light with different wavelengths; this recombination by defects is called the Shockley-Read-Hall recombination. In the case of films composed of $\mathrm{PbS} \mathrm{NCs}$ and $\mathrm{Alq}_{3}$ layer, higher emission intensity was observed than for the films without the $\mathrm{Alq}_{3}$ layer. Insertion of the $\mathrm{Alq}_{3}$ layer containing organic molecules induced multiple excitons generation from Förster energy between the LUMO represented by $\left(\mathrm{Alq}_{3}\right)$ and the HOMO represented by (FTO); this created improved HWLED efficiency [25-27]. Fig. 5 shows the presence of blue, green, and red peaks that have been detected. Their combination provides white light.

In the white light field, the emission light performance recorded in the EL spectrum is confirmed by the photograph of white light released from the FTO/PEDOT: PSS/PbS/Alq $3 / \mathrm{Ni}$ HWLED as shown in Fig. 6. The light intensity from the HWLEDs is quite strong.
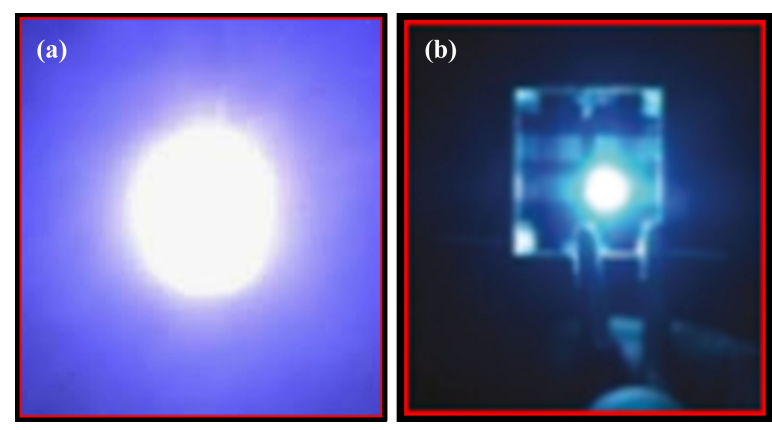

Fig. 6. Images of white light generation from FTO/PEDOT: PSS/PbS/Alq 3 /Ni HWLED.

Since the CIE colorimetry method describes the color of light, XYZ color matching functions sum up the spectrum of a given light. On the CIE colorimetry scheme, $\mathrm{X}, \mathrm{Y}$ and $\mathrm{Z}$ coordinates for chromaticity are located [28]:

$$
\begin{aligned}
& x=\frac{X+Y+Z}{X} \\
& y=\frac{X+Y+Z}{Y}
\end{aligned}
$$

The resulting white light color temperature (CCT) that might be determined by using the McCamy's approximation algorithm in the $x-y-$ chromaticity-coordinates [29]:

$$
C C T=-449 n^{3}+3525 n^{2}-6823 n+5520.33
$$


where

$$
n=\frac{x-0.332}{y-1858}
$$

The illustration of the HWLED in Fig. 6 indicates the output light of the EL emission in the location of white light and the strength of the output light is very high. Fig. 7 shows tristimulus coordinates (color coordinates) of the HWLEDs.

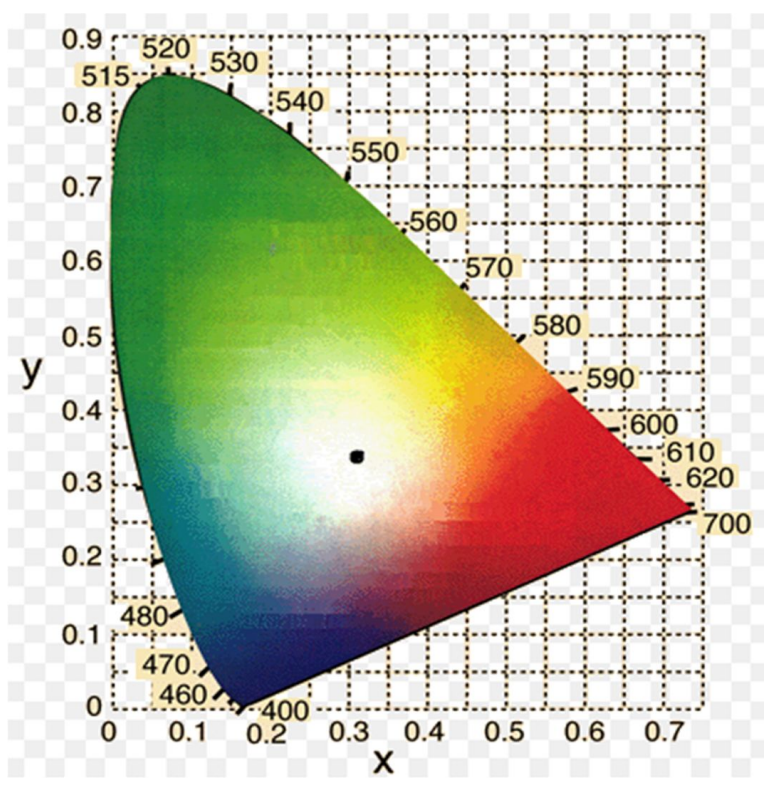

Fig. 7. The CIE 1931 chromaticity coordinates scheme for the HWLEDs.

The chromaticity coordinates values $(\mathrm{x}=0.31, \mathrm{y}=0.34)$ show that the hybrid FTO/PEDOT:PSS/PbS/Alq $3 / \mathrm{Ni}$ device has a color temperature of around $6250 \mathrm{~K}$. This means that the multiple excitons produced from the reaction of charge carriers between the PbS NCs and organic polymer increased the illumination of the output light in the center of the white light at higher temperatures. Therefore, the high temperature resulting in increased strength and efficiency of the output light confirmed that the white light from the HWLEDs system has been shifted towards the center of the white region in the coordinate system of chromaticity.

\section{Conclusion}

In this work, the colloidal $\mathrm{PbS} \mathrm{NCs}$ featuring narrow bandwidth emission and high fluorescence offers promising properties for their use in HWLEDs to produce a large color spectrum. Organic polymers PEDOT: PSS and $\mathrm{Alq}_{3}$ including organic molecules layers led to improving the efficiency of HWLEDs. The PbS NCs have also shown multiple exciton generation (MEG); two electronhole pairs (excitons) are formed by the absorption of one high-energy photon; this phenomenon has been used to improve the efficiency of the photoconversion of HWLEDs. The HWLEDs fabricated from semiconductor material (PbS NCs) with hole-injecting organic polymer (PEDOT: PSS with PMMA) and electron-injecting $\left(\mathrm{Alq}_{3}\right)$ proved to be efficient in generation of white light with high intensity.

\section{References}

[1] Yanqin M., Xiaozhen W., Long G., Kexiang W., Bo Z., Zhongqiang W., Bin Z., Hua W., YuCHENG W., BIngshe X., Nanophotonics, 8 (2019), 1783.

[2] Ekaterina N., Julia F., Sandra G., Adv. Funct. Mater, (2020), 1.

[3] Akeel M. K., NHC, 29 (2020), 2297.

[4] Daekyung K., Mareddi B. K., Changhee S., Hongsik P., Jonghoo P., Appl. Sci., 9 (2019), 1.

[5] Ashour M., Mohamed R., Mohamed S., RSC Adv., $10,(2020), 14458$.

[6] Vanessa W., Vladimir B., Nano Rev., 1 (2010), 5202.

[7] Philippe B., ZiQi L., Mark W., J. Phys. Chem. Lett., 10 (2019), 5897.

[8] AKEEL M.K., JMNM, 29 (2017), 2297.

[9] XiaO P., Huang J., Yicong Y., Baiquan L., Molecules, 24 (2019), 1.

[10] Tzu-Ming L., Joao C., Tomasz L., Artur B., Chin-Chia H., NPG Asia Mater, 8 (2016), 295.

[11] Do Y., Tzung-Han L., Jae W., Jesse R., Franky S., Sci. Rep., 4 (2015), 1.

[12] Yue M., YU Z., William W., J. Mater. Chem. C, 7 (2019), 13662.

[13] Xiulei S., Song C., Meng-Yao L., BiaO H., Guozhen Z., Ran C., MingXi Z., Nano Res., 13 (2020), 2239.

[14] Kroupa D.M., Pach G.F., Vörös M., Giberti F., Chernomordik B.D., Crisp R.W., NoziK A.J., Johnson J.C., Singh R., Klimov V.I., Galli G., BEARD M.C., ACS Nano, 12 (2018), 10084.

[15] Smith C., Binks D., Nanomaterials, 4 (2014), 19. 
[16] Kershaw S.V., Rogach A.L., Materials, 10 (9) (2017), 1095.

[17] So-Yeon P., Younghoon K., Sohee J., Dong H., Gill S., Hyun S., Chem. Phys. Chem., 20 (2019), 2657.

[18] Chen Q., Marco de N., Yang Y., Song T., Chen C., ZhaO H., Hong Z., Zhou H., YANG Y., Nano Today, 10 (2015), 355.

[19] Zhang F., Lu H., Tong J., Berry J. J., Beard M. C., ZHU K., Energy Environ. Sci., 13 (2020), 1154.

[20] Wu J., Chen S., ACS Appl. Mater. Interfaces, 10 (2018), 4851.

[21] Xu J., Miao Y., Zheng J., Wang H., Yang Y., LiU X., Nanoscale, 10 (2018), 11211.

[22] Shi H., Deng L., Chen S., Xu Y., Zhao X., Cheng F., HUANG W., AIP Adv., 4 (2014), 047110.

[23] Nishihara T., Tahara H., OKano M., Ono M., Kanemitsu Y., J. Phys. Chem. Lett., 8 (2015), 1327.
[24] Yang Y., Rodriguez-Cordoba W., Lian T., Nano Lett., 12 (2012), 4235.

[25] Cunningham P.D., Boercker J.E., Foos E E., Lumb M.P., Smith A.R., Tischler J.G., Melinger J.S., Nano Lett., 11 (2011), 3476.

[26] Cunningham P.D., Boercker J.E., Foos E E., Lumb M.P., SMith A.R., Tischler J.G., Melinger J.S., Sci. Rep., 5 (2015), 1.

[27] Cao W., Zhang Z., Patterson R., Lin Y., Wen X., Veetil B.P., Zhang P., Zhang Q., Shrestha S., Conibeer G., Huang S., RSC Adv., 6 (2016), 90846.

[28] Arthur D., Color Res. Appl., 29 (2004), 267.

[29] Li C., Melgosa C., Ruan X., Zhang Y., Ma L., Xiao K. Luo M.R., Opt. Soc. Am., 24 (2016), 13.

Received 2020-08-27 Accepted 2020-11-03 